\title{
Super-hydrophobic and self-healing Mg-Al layered double hydroxides/silane composite coatings on the Mg alloy surface with a long-term anti-corrosion
}

\section{lifetime}

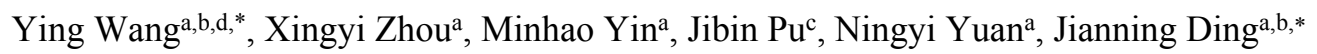

${ }^{a}$ School of Materials Science and Engineering, Jiangsu Collaborative Innovation Center for Photovoltic Science and Engineering, Jiangsu Province Cultivation base for State Key Laboratory of Photovoltaic Science and Technology, Changzhou University, Changzhou 213164, China

${ }^{\mathrm{b}}$ Micro/Nano Science and Technology Center, Jiangsu University, Zhenjiang 212013, China

${ }^{\mathrm{c}}$ Key Laboratory of Marine Materials and Related Technologies, Zhejiang Key Laboratory of Marine Materials and Protective Technologies, Ningbo Institute of Materials Technology and Engineering, Chinese Academy of Sciences, Ningbo 315201, China.

d State Key Laboratory of Solid Lubrication, Lanzhou Institute of Chemical Physics, Chinese Academy of Sciences, Lanzhou 730000, PR China

${ }^{*}$ Corresponding authors.

E-mail address: ywang@,cczu.edu.cn (Y. Wang), dingjn@,cczu.edu.cn (J.N. Ding). 


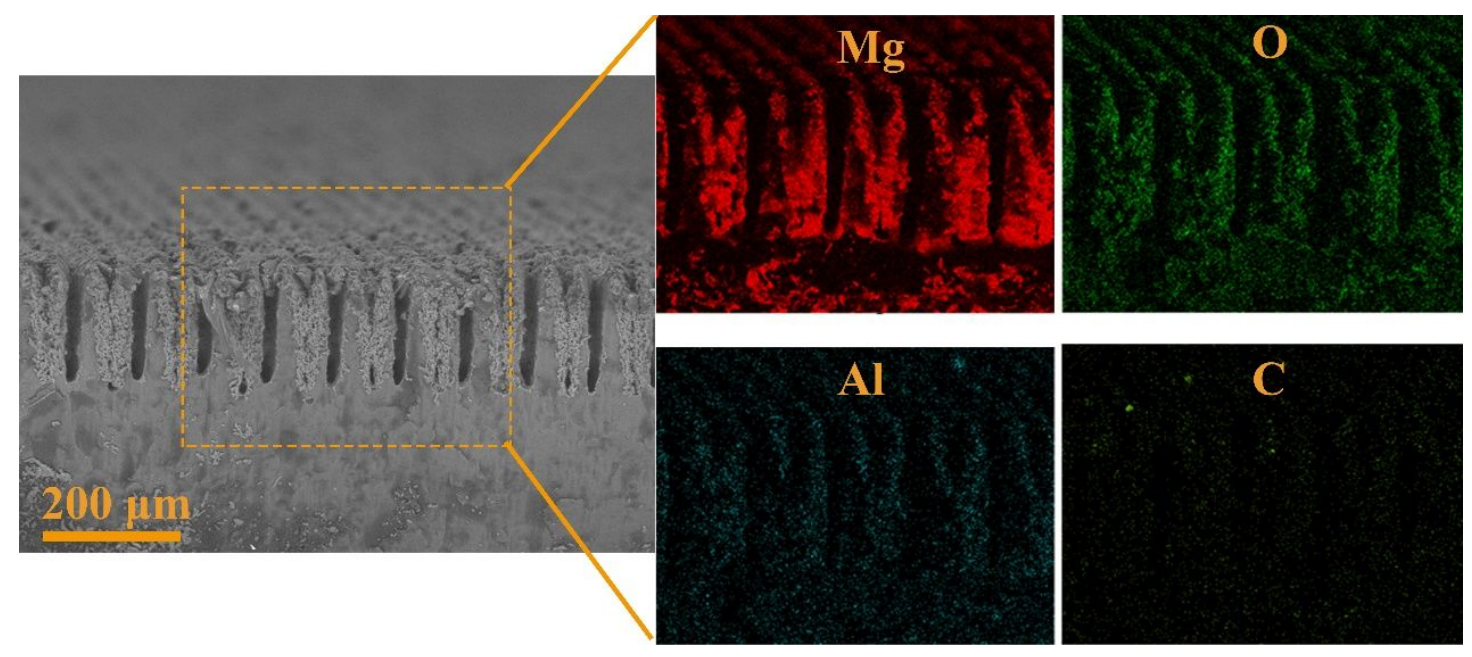

Figure S1. The SEM and EDS cross-sectional images of L.

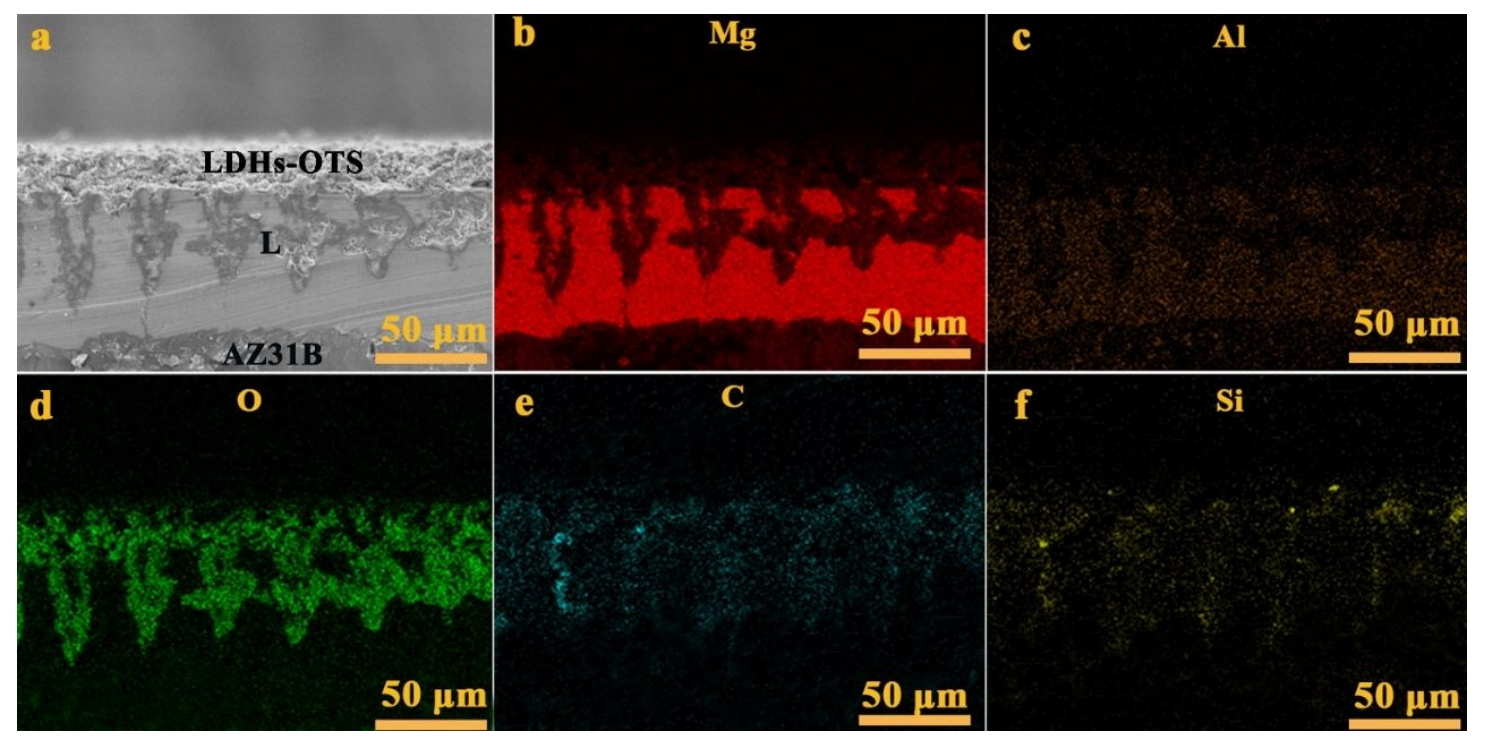

Figure S2. The SEM (a) and EDS (b, c, d, e, f) cross-sectional images of L-LDHs-OTS.

Table S1. Tafel fitting results of different samples.

\begin{tabular}{lccc}
\hline Sample & $E_{\text {corr }}(\mathrm{V} / \mathrm{SCE})$ & $i_{\text {corr }}\left(\mathrm{A} / \mathrm{cm}^{2}\right)$ & $P_{i}(\mathrm{~mm} / \mathrm{y})$ \\
\hline Bare & -1.517 & $4.04 \times 10^{-5}$ & 0.92 \\
$\mathrm{~L}$ & -1.457 & $6.05 \times 10^{-6}$ & 0.14
\end{tabular}



L-LDHs
$-1.214$
$4.58 \times 10^{-8}$
$1.04 \times 10^{-3}$
L-LDHs-OTS
$-0.876$
$1.08 \times 10^{-10}$
$2.29 \times 10^{-6}$

Table S2. Comparison of corrosion resistance of LDHs composite coatings (Electrolyte: 3.5 wt.\% NaCl).

\begin{tabular}{lcccc}
\hline Sample & Substrate & $\begin{array}{c}\text { Substrate } \\
i_{\text {corr }}\left(\mathrm{A} / \mathrm{cm}^{2}\right)\end{array}$ & $\begin{array}{c}\text { Coating } \\
i_{\text {corr }}\left(\mathrm{A} / \mathrm{cm}^{2}\right)\end{array}$ & Ref. \\
\hline Mg Al-NO 3 LDHs & AZ91D & $3.05 \times 10^{-6}$ & $3.36 \times 10^{-7}$ & {$[35]$} \\
Mg Al-LDHs/anodic & AZ31B & $3.27 \times 10^{-5}$ & $1.18 \times 10^{-7}$ & {$[36]$} \\
Mg Al-ASP LDHs & AZ31B & $2.703 \times 10^{-5}$ & $2.24 \times 10^{-7}$ & {$[16]$} \\
L-LDHs (this work) & AZ31B & $4.04 \times 10^{-5}$ & $4.58 \times 10^{-8}$ & $/$ \\
L-LDHs-OTS (this work) & AZ31B & $4.04 \times 10^{-5}$ & $1.08 \times 10^{-10}$ & $/$ \\
\hline
\end{tabular}

Table S3. Tafel fitting results of L-LDHs-OTS immersed for different times.

\begin{tabular}{lccc}
\hline Sample & $E_{\text {corr }}(\mathrm{V} / \mathrm{SCE})$ & $i_{\text {corr }}\left(\mathrm{A} / \mathrm{cm}^{2}\right)$ & $P_{i}(\mathrm{~mm} / \mathrm{y})$ \\
\hline 0 day & -0.876 & $1.08 \times 10^{-10}$ & $2.29 \times 10^{-6}$ \\
4 days & -1.12 & $3.14 \times 10^{-10}$ & $7.17 \times 10^{-6}$ \\
8 days & -1.18 & $7.54 \times 10^{-10}$ & $1.73 \times 10^{-5}$ \\
12 days & -1.07 & $8.66 \times 10^{-9}$ & $1.98 \times 10^{-4}$ \\
16 days & -0.56 & $9.07 \times 10^{-10}$ & $2.07 \times 10^{-5}$ \\
20 days & -0.68 & $9.38 \times 10^{-10}$ & $2.14 \times 10^{-5}$ \\
\hline
\end{tabular}

